\title{
Milena Matusik
}

\section{IMPLICIT DIFFERENCE SCHEMES FOR QUASILINEAR PARABOLIC FUNCTIONAL EQUATIONS}

\begin{abstract}
We present a new class of numerical methods for quasilinear parabolic functional differential equations with initial boundary conditions of the Robin type. The numerical methods are difference schemes which are implicit with respect to time variable. We give a complete convergence analysis for the methods and we show that the new methods are considerable better than the explicit schemes. The proof of the stability is based on a comparison technique with nonlinear estimates of the Perron type for given functions with respect to functional variables. Results obtained in the paper can be applied to differential equations with deviated variables and to differential integral problems.
\end{abstract}

\section{Introduction}

Difference schemes for quasilinear parabolic functional differential equations consist in replacing partial derivatives with difference operators. Moreover, because differential equations contain functional variables, some interpolating operators are needed. This leads to functional difference equations which satisfy consistency conditions on classical solutions of original problems. The main task in these considerations is to find difference approximations of functional differential equations which are stable.

From the numerous literature concerning explicit difference methods we mention the papers [1], [13], where quasilinear parabolic functional differential equations with initial boundary conditions of the Dirichlet type were considered. Parabolic equations with initial boundary conditions of the Neumann type were investigated in [2], [14].

The papers [3], [5] initiated the theory of implicit difference schemes for quasilinear equations. Initial boundary value problems of the Dirichlet type were investigated in [3]. Numerical treatment of initial boundary value problems of the Neumann type can be found in [5], [6]. A method of dif-

2000 Mathematics Subject Classification: 35R10, 65M12.

Key words and phrases: quasilinear parabolic functional differential equations, implicit difference methods, nonlinear estimates of the Perron type. 
ference inequalities and theorems on recurrent inequalities are used in the investigations of the stability of implicit difference schemes.

Monotone iterative methods and implicit difference schemes for computing approximate solutions to parabolic equations with time delays were studied in [7]-[10], [15].

The aim of the paper is to present a new class of numerical methods for quasilinear parabolic functional differential equations with initial boundary conditions of the Robin type. The numerical methods are difference schemes which are implicit with respect to the time variable. We give sufficient conditions for the convergence of the methods and we show by examples that the new methods are considerably better than classical schemes.

The proof of the convergence is based on comparison technique with nonlinear estimates of the Perron type with respect to the functional variables.

Now, we formulate our functional differential problems. For any metric spaces $X$ and $Y$ we denote by $C(X, Y)$ the class of all continuous functions defined on $X$ and taking values in $Y$. We will use vectorial inequalities with the understanding that the same inequalities hold between their corresponding components. Write

$$
E_{0}=\left[-b_{0}, 0\right] \times[-b, b], \quad E=[0, a] \times[-b, b],
$$

where $a>0, b_{0} \in \mathbb{R}_{+}, \mathbb{R}_{+}=[0,+\infty)$ and $b=\left(b_{1}, \ldots, b_{n}\right), b_{i}>0$ for $i=1, \ldots, n$. Set $r_{0}=b_{0}+a, r=2 b$ and $B=\left[-r_{0}, 0\right] \times[-r, r], \Sigma=$ $\left[-r_{0}, a\right] \times[-b-r, b+r]$. For a function $z: \Sigma \rightarrow \mathbb{R}$ and for a point $(t, x) \in E$ we define a function $z_{(t, x)}: B \rightarrow \mathbb{R}$ by

$$
z_{(t, x)}(\tau, y)=z(t+\tau, x+y), \quad(\tau, y) \in B .
$$

For $(t, x) \in E$ we put

$$
D[t, x]=\left\{(\tau, y) \in \mathbb{R}^{1+n}: \tau \leq 0, \quad(t+\tau, x+y) \in E_{0} \cup E\right\} .
$$

It is clear that $D[t, x]=\left[-b_{0}-t, 0\right] \times[-b-x, b-x]$ and $D[t, x] \subset B$ for $(t, x) \in E$. Let $M_{n \times n}$ be the class of all $n \times n$ matrices with real elements. Write $\Xi=E \times C(B, \mathbb{R})$ and suppose that $\mathbf{f}: \Xi \rightarrow M_{n \times n}, \mathbf{f}=\left[f_{i j}\right]_{i, j=1, \ldots, n}$, $\mathrm{g}: \Xi \rightarrow \mathbb{R}^{n}, \mathbf{g}=\left(g_{1}, \ldots, g_{n}\right) G: \Xi \rightarrow \mathbb{R}$ are given functions. We will say that $\mathbf{f}, \mathbf{g}$ and $G: \Xi \rightarrow \mathbb{R}$ satisfy the condition $(V)$ if for each $(t, x, w) \in \Xi$ and $\tilde{w} \in C(B, \mathbb{R})$ such that $w(\tau, y)=\tilde{w}(\tau, y)$ for $(\tau, y) \in D[t, x]$, we have $\mathbf{f}(t, x, w)=\mathbf{f}(t, x, \tilde{w}), \mathbf{g}(t, x, w)=\mathbf{g}(t, x, \tilde{w})$ and $G(t, x, w)=G(t, x, \tilde{w})$. Note that the condition $(V)$ means that the value of $\mathbf{f}, \mathbf{g}$ and $G$ at the point $(t, x, w) \in \Xi$ depends on $(t, x)$ and on the restriction of $w$ to the set $D[t, x]$ only. Let us denote by $z$ an unknown function of the variables $(t, x), x=$ $\left(x_{1}, \ldots, x_{n}\right)$. We consider the functional differential equation 


$$
\begin{aligned}
\partial_{t} z(t, x)= & \sum_{i, j=1}^{n} f_{i j}\left(t, x, z_{(t, x)}\right) \partial_{x_{i} x_{j}} z(t, x) \\
& +\sum_{i=1}^{n} g_{i}\left(t, x, z_{(t, x)}\right) \partial_{x_{i}} z(t, x)+G\left(t, x, z_{(t, x)}\right)
\end{aligned}
$$

where $\partial_{x} z=\left(\partial_{x_{1}} z, \ldots, \partial_{x_{n}} z\right), \partial_{x x} z=\left[\partial_{x_{i} x_{j}} z\right]_{i, j=1, \ldots, n}$. We assume that $\mathbf{f}, \mathbf{g}$ and $G$ satisfy the condition $(V)$ and we consider classical solutions of (1.1). Now we formulate initial boundary conditions for (1.1). Write

$$
S_{i}=\left\{x \in[-b, b]: x_{i}=b_{i}\right\}, \quad S_{n+i}=\left\{x \in[-b, b]: x_{i}=-b_{i}\right\}, i=1, \ldots, n
$$

and

$$
Q_{1}^{+}=S_{1}, \quad Q_{i}^{+}=S_{i} \backslash \bigcup_{j=1}^{i-1} S_{j}, \quad Q_{i}^{-}=S_{n+i} \backslash \bigcup_{j=1}^{n+i-1} S_{j}, \quad i=1, \ldots, n .
$$

Set

$$
\partial_{0} E_{i}^{+}=[0, a) \times Q_{i}^{+}, \quad \partial_{0} E_{i}^{-}=[0, a) \times Q_{i}^{-}, i-1, \ldots, n
$$

and

$$
\partial_{0} E=\bigcup_{i=1}^{n}\left(\partial_{0} E_{i}^{+} \cup \partial_{0} E_{i}^{-}\right) \text {. }
$$

Suppose that $\beta, \gamma, \Psi: \partial_{0} E \rightarrow \mathbb{R}, \psi: E_{0} \rightarrow \mathbb{R}$ are given functions. The following initial boundary conditions are associated with (1.1):

$$
\begin{gathered}
z(t, x)=\psi(t, x) \quad \text { on } E_{0}, \\
\beta(t, x) z(t, x)+\gamma(t, x) \partial_{x_{i}} z(t, x)=\Psi(t, x) \quad \text { on } \partial_{0} E_{i}^{+}, i=1, \ldots, n, \\
\beta(t, x) z(t, x)-\gamma(t, x) \partial_{x_{i}} z(t, x)=\Psi(t, x) \quad \text { on } \partial_{0} E_{i}^{-}, i=1, \ldots, n .
\end{gathered}
$$

A function $z: E_{0} \cup E \rightarrow \mathbb{R}$ will be called the function of class $C_{*}$ if $z$ is continuous on $E_{0} \cup E$, the partial derivatives $\partial_{t} z, \partial_{x} z=\left(\partial_{x_{1}} z, \ldots, \partial_{x_{n}} z\right)$, $\partial_{x x} z=\left[\partial_{x_{i} x_{j}} z\right]_{i, j=1, \ldots, n}$ exist on $E$ and the functions $\partial_{t} z, \partial_{x} z, \partial_{x x} z$ are continuous on $E$. We consider solutions of (1.1)-(1.4) of class $C_{*}$.

For spaces $X$ and $Y$ we denote by $\mathcal{F}(X, Y)$ the class of all functions defined on $X$ and taking values in $Y$. Solutions of difference functional equations are elements of the space $\mathcal{F}\left(E_{0 . \mathbf{h}} \cup E_{\mathbf{h}}, \mathbb{R}\right)$. Since equation (1.1) contains the functional variable $z_{(t, x)}$ which is an element of the space $C(D[t, x], \mathbb{R})$, we need an interpolating operator $T_{\mathbf{h}}: \mathcal{F}\left(B_{\mathbf{h}}, \mathbb{R}\right) \rightarrow C(B, \mathbb{R})$. For a function $z \in \Sigma_{\mathbf{h}} \rightarrow \mathbb{R}$ and for a point $\left(t^{(r)}, x^{(m)}\right) \in E_{\mathbf{h}}$ we define a function $z_{[r, m]}: B_{\mathbf{h}} \rightarrow \mathbb{R}$ by

$$
z_{[r, m]}(\tau, y)=z\left(t^{(r)}+\tau, x^{(m)}+y\right), \quad(\tau, y) \in B_{\mathbf{h}} .
$$

Let $\mathbb{N}$ and $\mathbb{Z}$ be the sets of natural numbers and integers, respectively. We define a mesh in $\mathbb{R}^{1+n}$ in the following way. Let $\mathbf{h}=\left(h_{0}, h\right), h=\left(h_{1}, \ldots, h_{n}\right)$, 
stand for steps of the mesh. For $(r, m) \in \mathbb{Z}^{1+n}, m=\left(m_{1}, \ldots, m_{n}\right)$ we define nodal points as follows

$$
t^{(r)}=r h_{0}, \quad x^{(m)}=\left(m_{1} h_{1}, \ldots, m_{n} h_{n}\right)=\left(x_{1}^{\left(m_{1}\right)}, \ldots, x_{n}^{\left(m_{n}\right)}\right) .
$$

Let us denote by $\mathbb{H}$ the set of all $\mathbf{h}$ for which there exist $\left(M_{1}, \ldots, M_{n}\right)=$ $M \in \mathbb{Z}^{n}$ and $M_{0} \in \mathbb{Z}$ such that $M_{i} h_{i}=b_{i}$ for $i=1, \ldots, n, M_{0} h_{0}=b_{0}$. Let $K \in \mathbb{N}$ be defined by relations $K h_{0} \leq a<(K+1) h_{0}$. For $\mathbf{h} \in \mathbb{H}$ we put

$$
\mathbb{R}_{\mathbf{h}}^{1+n}=\left\{\left(t^{(r)}, x^{(m)}\right):(r, m) \in \mathbb{Z}^{1+n}\right\}
$$

and

$$
\begin{gathered}
E_{0 . \mathbf{h}}=E_{0} \cap \mathbb{R}_{\mathbf{h}}^{1+n}, \quad E_{\mathbf{h}}=E \cap \mathbb{R}_{\mathbf{h}}^{1+n}, \quad B_{\mathbf{h}}=B \cap \mathbb{R}_{\mathbf{h}}^{1+n}, \\
\partial_{0} E_{\mathbf{h} . i}^{+}=\partial_{0} E_{i}^{+} \cap \mathbb{R}_{\mathbf{h}}^{1+n}, \quad \partial_{0} E_{\mathbf{h} . i}^{-}=\partial_{0} E_{i}^{+} \cap \mathbb{R}_{\mathbf{h}}^{1+n}, \quad \Xi_{\mathbf{h}}=E_{\mathbf{h}} \times C\left(B_{\mathbf{h}}, \mathbb{R}\right) .
\end{gathered}
$$

Suppose that $\psi_{\mathbf{h}}: E_{0 . \mathbf{h}} \rightarrow \mathbb{R}$ and $\Psi_{\mathbf{h}}: \partial_{0} E_{\mathbf{h}} \rightarrow \mathbb{R}$ are given functions. We consider the difference functional equation corresponding to (1.1).

$$
\begin{aligned}
\delta_{0} z^{(r, m)}= & \sum_{i, j=1}^{n} f_{i j}\left(t^{(r)}, x^{(m)}, T_{\mathbf{h}} z_{[r, m]}\right) \delta_{i j} z^{(r+1, m)} \\
& +\sum_{i=1}^{n} g_{i}\left(t^{(r)}, x^{(m)}, T_{\mathbf{h}} z_{[r, m]}\right) \delta_{i} z^{(r+1, m)} \\
& +G\left(t^{(r)}, x^{(m)}, T_{\mathbf{h}} z_{[r, m]}\right),
\end{aligned}
$$

where the difference operators $\delta_{0}, \delta=\left(\delta_{1}, \ldots, \delta_{n}\right), \delta^{(2)}=\left[\delta_{i j}\right]_{i, j=1, \ldots, n}$ are defined in the following way. Let $e_{i}=(0, \ldots, 0,1,0, \ldots, 0) \in \mathbb{R}^{n}$ with 1 standing on the $i$-th place. Write $J=\{(i, j): i, j=1, \ldots, n, i \neq j\}$. For a function $z: E_{0 . \mathbf{h}} \cup E_{\mathbf{h}} \rightarrow \mathbb{R}$ and for a point $\left(t^{(r)}, x^{(m)}\right) \in E_{\mathbf{h}}$ we define the sets

$$
\begin{aligned}
& J_{-}^{(r, m)}[z]=\left\{(i, j) \in J: f_{i j}\left(t^{(r)}, x^{(m)}, T_{\mathbf{h}} z_{[r, m]}\right) \leq 0\right\}, \\
& J_{+}^{(r, m)}[z]=J \backslash J_{-}^{(r, m)}[z] .
\end{aligned}
$$

Let $P^{(r, m)}[z]=\left(t^{(r)}, x^{(m)}, T_{\mathbf{h}} z_{[r, m]}\right)$. Given $z \in \mathcal{F}\left(E_{0 . \mathbf{h}} \cup E_{\mathbf{h}}, \mathbb{R}\right)$ and $(r, m) \in$ $\mathbb{Z}^{1+n}, 0 \leq r \leq K-1,-(M-1) \leq m \leq M-1$, where $M-1=\left(M_{1}-\right.$ $\left.1, \ldots, M_{n}-1\right)$. Write

$$
\begin{aligned}
\delta_{0} z^{(r, m)} & =\frac{1}{h_{0}}\left[z^{(r+1, m)}-z^{(r, m)}\right], \\
\delta_{i}^{+} z^{(r, m)} & =\frac{1}{h_{i}}\left[z^{\left(r, m+e_{i}\right)}-z^{(r, m)}\right], \\
\delta_{i}^{-} z^{(r, m)} & =\frac{1}{h_{i}}\left[z^{(r, m)}-z^{\left(r, m-e_{i}\right)}\right], \quad i=1, \ldots, n, \\
\delta z^{(r, m)} & =\left(\delta_{1} z^{(r, m)}, \ldots, \delta_{n} z^{(r, m)}\right)
\end{aligned}
$$


where

$$
\delta_{i} z^{(r, m)}=\frac{1}{2}\left[\delta_{i}^{+} z^{(r, m)}+\delta_{i}^{-} z^{(r, m)}\right], i=1, \ldots, n .
$$

The difference operator $\delta^{(2)}=\left[\delta_{i j}\right]_{i, j=1, \ldots, n}$, is defined in the following way:

$$
\delta_{i i}^{(2)} z^{(r, m)}=\delta_{i}^{+} \delta_{i}^{-} z^{(r, m)} \quad \text { for } i=1, \ldots, n
$$

and

$$
\begin{array}{ll}
\delta_{i j}^{(2)} z^{(r, m)}=\frac{1}{2}\left[\delta_{i}^{+} \delta_{j}^{-} z^{(r, m)}+\delta_{i}^{-} \delta_{j}^{+} z^{(r, m)}\right], & \text { if }(i, j) \in J_{-}^{(r, m)}[z], \\
\delta_{i j}^{(2)} z^{(r, m)}=\frac{1}{2}\left[\delta_{i}^{+} \delta_{j}^{+} z^{(r, m)}+\delta_{i}^{-} \delta_{j}^{-} z^{(r, m)}\right], & \text { if }(i, j) \in J_{+}^{(r, m)}[z] .
\end{array}
$$

Set

$$
\begin{array}{ll}
\Lambda_{\mathbf{h} . i}^{+}[z]^{(r, m)}=\beta^{(r, m)} z^{(r, m)}+\gamma^{(r, m)} \delta_{i}^{-} z^{(r, m)} & \text { on } \partial_{0} E_{\mathbf{h} . i}^{+}, \\
\Lambda_{\mathbf{h} . i}^{-}[z]^{(r, m)}=\beta^{(r, m)} z^{(r, m)}-\gamma^{(r, m)} \delta_{i}^{+} z^{(r, m)} & \text { on } \partial_{0} E_{\mathbf{h} . i}^{-}
\end{array}
$$

where $i=1, \ldots, n$. Write

$$
\partial_{0} E_{\mathbf{h}}^{+}=\bigcup_{i=1}^{n} \partial_{0} E_{\mathbf{h} . i}^{+}, \quad \partial_{0} E_{\mathbf{h}}^{-}=\bigcup_{i=1}^{n} \partial_{0} E_{\mathbf{h} . i}^{-}, \quad \partial_{0} E_{\mathbf{h}}=\partial_{0} E_{\mathbf{h}}^{+} \cup \partial_{0} E_{\mathbf{h}}^{-} .
$$

For a function $z: E_{0 . \mathbf{h}} \cup E_{\mathbf{h}} \rightarrow \mathbb{R}$ we define a function $\Lambda_{\mathbf{h}}[z]: \partial_{0} E_{\mathbf{h}} \rightarrow \mathbb{R}$ in the following way:

$$
\begin{aligned}
& \Lambda_{\mathbf{h}}[z]^{(r, m)}=\Lambda_{\mathbf{h} . i}^{+}[z]^{(r, m)} \quad \text { if } \quad\left(t^{(r)}, x^{(m)}\right) \in \partial_{0} E_{\mathbf{h} . i}^{+}, \\
& \Lambda_{\mathbf{h}}[z]^{(r, m)}=\Lambda_{\mathbf{h}}^{-}[z]^{(r, m)} \quad \text { if } \quad\left(t^{(r)}, x^{(m)}\right) \in \partial_{0} E_{\mathbf{h} . i}^{-} .
\end{aligned}
$$

The following initial boundary conditions are associated with (1.5)

$$
\begin{aligned}
z^{(r, m)}=\psi_{\mathbf{h}}^{(r, m)} & \text { on } E_{0 . \mathbf{h}}, \\
\Lambda_{\mathbf{h}[z]^{(r, m)}}=\Psi_{\mathbf{h}}^{(r, m)} & \text { on } \partial_{0} E_{\mathbf{h} .} .
\end{aligned}
$$

Our motivations for the construction of implicit difference schemes are the following. Two type assumptions are needed in theorems on the convergence of the explicit difference methods generated by (1.1)-(1.4). The first type conditions concern regularity of $F$. The first type concern the regularity of $\mathbf{f}, \mathbf{g}$ and $G$. It is assumed that these functions are continuous and bounded on $\Xi$ and satisfy nonlinear estimates of the Perron type with respect to the functional variable. The second type conditions concern the mesh. It is required that difference schemes satisfy the condition

$$
1-2 h_{0} \sum_{i=1}^{n} \frac{1}{h_{i}^{2}} f_{i i}(t, x, w)+h_{0} \sum_{\substack{j=1 \\ j \neq i}}^{n} \frac{1}{h_{i} h_{j}}\left|f_{i j}(t, x, w)\right| \geq 0,(t, x, w) \in \Xi,
$$


(see [1], [13]) where $h_{0}$ and $\left(h_{1}, \ldots, h_{n}\right)$ are steps of the mesh with respect to $t$ and $\left(x_{1}, \ldots, x_{n}\right)$ respectively. It is clear that strong assumptions on relations between $h_{0}$ and $\left(h_{1}, \ldots, h_{n}\right)$ are required in (1.13). We show that there are difference methods for (1.1)-(1.4) which are convergent and assumption (1.13) is omitted.

The authors of the papers [1]-[3], [5], [6] have assumed that given functions satisfy the Lipschitz condition or satisfy nonlinear estimates of the Perron type with respect to the functional variable, and these conditions are global. We assume nonlinear estimates of the Perron type and suitable estimates are local with respect to functional variables. It is clear that there are differential equations with deviated variables and differential integral equations such that local estimates of the Perron type hold and global inequalities are not satisfied.

We use in the paper general ideas for finite difference equations which were introduced in the monographs [4], [11], [12].

\section{Solutions of functional differential and difference problems}

We first construct estimates of solutions to (1.1)-(1.4). For $W \in M_{n \times n}$ and $x \in \mathbb{R}^{n}$, where $W=\left[w_{i j}\right]_{i, j=1, \ldots, n}, x=\left(x_{1}, \ldots, x_{n}\right)$, we define

$$
\|W\|_{[n \times n]}=\sum_{i, j=1}^{n}\left|w_{i j}\right|, \quad\|x\|_{[n]}=\sum_{i=1}^{n}\left|x_{i}\right| .
$$

For functions $z \in C\left(E_{0} \cup E, \mathbb{R}\right), u \in \mathcal{F}\left(E_{0 . \mathbf{h}} \cup E_{\mathbf{h}}, \mathbb{R}\right)$ we define the semi-norms

$$
\begin{gathered}
\|z\|_{t}=\max \left\{|z(\tau, x)|:(\tau, x) \in\left(E_{0} \cup E\right) \cap\left(\left[-b_{0}, t\right] \times \mathbb{R}^{n}\right)\right\}, \\
\|u\|_{\mathbf{h} . r}=\max \left\{|z(\tau, x)|:(\tau, x) \in\left(E_{0 . \mathbf{h}} \cup E_{\mathbf{h}}\right) \cap\left(\left[-b_{0}, t^{(r)}\right] \times \mathbb{R}^{n}\right)\right\}, \quad 0 \leq r \leq K .
\end{gathered}
$$

Assumption $\mathbf{H}[\varrho]$. The function $\varrho:[0, a] \times \mathbb{R}_{+} \rightarrow \mathbb{R}_{+}$is continuous and it is nondecreasing with respect to both variables and for each $\eta \in \mathbb{R}_{+}$the maximal solution of the Cauchy problem

$$
\omega^{\prime}(t)=\varrho(t, \omega(t)), \quad \omega(0)=\eta
$$

is defined on $[0, a]$.

Assumption $\mathbf{H}_{0}[\mathbf{f}, \mathbf{g}, G]$. The functions $\mathbf{f}: \Xi \rightarrow M_{n \times n}, \mathbf{g}: \Xi \rightarrow \mathbb{R}^{n}$, $G: \Xi \rightarrow \mathbb{R}$ are continuous and they satisfy the condition $(V)$ and

1) the matrix $\mathbf{f}$ is symmetric and for $(t, x, w) \in \Xi$ we have

$$
\sum_{i, j=1}^{n} f_{i j}(t, x, w) y_{i} y_{j} \geq 0, \quad \text { where } y=\left(y_{1}, \ldots, y_{n}\right) \in \mathbb{R}^{n},
$$

2) there is $\varrho:[0, a] \times \mathbb{R}_{+} \rightarrow \mathbb{R}_{+}$such that Assumption $\mathbf{H}[\varrho]$ is satisfied and

$$
|G(t, x, w)| \leq \varrho\left(t,\|w\|_{B}\right) \quad \text { on } \Xi
$$

is satisfied on $\Xi$, 
3) the functions $\beta: \partial_{0} E \rightarrow(0,+\infty), \gamma: \partial_{0} E \rightarrow \mathbb{R}_{+}$are continuous and the constant $\tilde{B}>0$ is defined by the relation $\beta(t, x) \geq \tilde{B}$ on $\partial_{0} E$,

4) the constant $\tilde{\eta}$ is defined by the relations

$$
|\psi(t, x)| \leq \tilde{\eta} \text { on } E_{0} \quad \text { and } \quad|\Psi(t, x)| \leq \tilde{B} \omega(t, \tilde{\eta}) \text { on } \partial_{0} E,
$$

where $\omega(\cdot, \tilde{\eta})$ is a maximal solution to problem $(2.1)$ with $\eta=\tilde{\eta}$.

Theorem 2.1. Suppose that Assumption $\mathbf{H}_{0}[\mathbf{f}, \mathbf{g}, G]$ is satisfied and $v$ is a solution of problem (1.1)-(1.4) and $v$ is of class $C_{*}$. Then

$$
|v(t, x)| \leq \omega(t, \tilde{\eta}) \quad \text { on } E .
$$

Proof. For $\varepsilon>0$ we denote by $\omega(\cdot, \tilde{\eta}, \varepsilon)$ the right hand maximal solution of the Cauchy problem

$$
\omega^{\prime}(t)=\varrho(t, \omega(t))+\varepsilon, \quad \omega(0)=\tilde{\eta}+\varepsilon .
$$

The solution $\omega(\cdot, \tilde{\eta}, \varepsilon)$ is defined on $[0, a]$ and $\lim _{\varepsilon \rightarrow 0} \omega(t, \tilde{\eta}, \varepsilon)=\omega(t, \tilde{\eta})$ uniformly on $[0, a]$. Write $\zeta(t)=\|v\|_{t}, t \in[0, a]$. We prove that

$$
\zeta(t)<\omega(t, \tilde{\eta}, \varepsilon) \text { for } t \in[0, a] .
$$

Suppose by contradiction that assertion (2.5) fails to be true. Then the set $\Sigma_{+}=\{t \in[0, a]: \zeta(t) \geq \omega(t, \tilde{\eta}, \varepsilon)\}$ is not empty. If we put $\tilde{t}=\min \Sigma_{+}$, from condition 4) of Assumption $\mathbf{H}_{0}[\mathbf{f}, \mathbf{g}, G]$ it is clear that $\tilde{t}>0$ and there exists $\tilde{x} \in[-b, b]$ such that

$$
\omega(\tilde{t}, \tilde{\eta}, \varepsilon)=\zeta(\tilde{t})=|v(\tilde{t}, \tilde{x})| .
$$

Then two possibilities can happen, either $(i) v(\tilde{t}, \tilde{x})=\omega(\tilde{t}, \tilde{\eta}, \varepsilon)$ or $(i i)$ $v(\tilde{t}, \tilde{x})=-\omega(\tilde{t}, \tilde{\eta}, \varepsilon)$. Let us consider the first case. We conclude from conditions 3) and 4) of Assumption $\mathbf{H}_{0}[\mathbf{f}, \mathbf{g}, G]$ that $(\tilde{t}, \tilde{x}) \notin \partial_{0} E$. Hence $(\tilde{t}, \tilde{x}) \in E \backslash \partial_{0} E$. We have that

$$
D_{-} \zeta(\tilde{t}) \geq \omega^{\prime}(\tilde{t}, \tilde{\eta}, \varepsilon) .
$$

On the other hand $\tilde{x} \in(-b, b)$, hence $\partial_{x} v(\tilde{t}, \tilde{x})=0$ and

$$
\sum_{i, j=1}^{n} \partial_{x_{i} x_{j}} v(\tilde{t}, \tilde{x}) \lambda_{i} \lambda_{j} \leq 0 \quad \text { for } \lambda \in \mathbb{R}^{n} .
$$

We have that

$$
\begin{aligned}
D_{-} \zeta(\tilde{t}) \leq \partial_{t} v(\tilde{t}, \tilde{x})= & \sum_{i, j=1}^{n} f_{i j}\left(\tilde{t}, \tilde{x}, v_{(\tilde{t}, \tilde{x})}\right) \partial_{x_{i} x_{j}} v(\tilde{t}, \tilde{x}) \\
& +\sum_{i=1}^{n} g_{i}\left(\tilde{t}, \tilde{x}, v_{(\tilde{t}, \tilde{x})}\right) \partial_{x_{i}} v(\tilde{t}, \tilde{x})+G\left(\tilde{t}, \tilde{x}, v_{(\tilde{t}, \tilde{x})}\right) \\
\leq & G\left(\tilde{t}, \tilde{x}, v_{(\tilde{t}, \tilde{x})}\right) \leq \varrho\left(\tilde{t},\|v\|_{\tilde{t}}\right)<\omega^{\prime}(\tilde{t}, \tilde{\eta}, \varepsilon),
\end{aligned}
$$


which contradicts (2.6). The case $v(\tilde{t}, \tilde{x})=-\omega(\tilde{t}, \tilde{\eta}, \varepsilon)$ can be treated in a similar way. Hence $\Sigma_{+}$is empty and inequality $(2.3)$ is proved on $E$. This completes the proof.

Assumption $\mathbf{H}[\mathbf{f}, \mathbf{g}, G]$. Assumption $\mathbf{H}_{0}[\mathbf{f}, \mathbf{g}, G]$ is satisfied and functions f, $g$ satisfy the inequality

$$
-\frac{1}{2}\left|g_{i}(P)\right|+\frac{1}{h_{i}} f_{i i}(P)-\sum_{\substack{j=1 \\ j \neq i}}^{n} \frac{1}{h_{j}}\left|f_{i j}(P)\right| \geq 0, \quad i=1, \ldots, n, \quad P \in \Xi .
$$

Set $P^{(r, m)}[z]=\left(t^{(r)}, x^{(m)}, T_{\mathbf{h}} z_{[r, m]}\right)$ and

$$
\begin{aligned}
F_{\mathbf{h}}[z]^{(r, m)}= & \sum_{i, j=1}^{n} f_{i j}\left(P^{(r, m)}[z]\right) \delta_{i j} z^{(r+1, m)} \\
& +\sum_{i=1}^{n} g_{i}\left(P^{(r, m)}[z]\right) \delta_{i} z^{(r+1, m)}+G\left(P^{(r, m)}[z]\right) .
\end{aligned}
$$

LemMa 2.1. Suppose that Assumption $\mathbf{H}[\mathbf{f}, \mathbf{g}, G]$ is satisfied and $z \in$ $\mathcal{F}\left(E_{0 . h} \cup E_{\boldsymbol{h}}, \mathbb{R}\right)$ and

$$
\mathcal{G}_{h}^{(r, m)}[w, z]=\sum_{i, j=1}^{n} f_{i j}\left(P^{(r, m)}[w]\right) \delta_{i j} z^{(r+1, m)}+\sum_{i=1}^{n} g_{i}\left(P^{(r, m)}[w]\right) \delta_{i} z^{(r+1, m)} .
$$

Then

$$
\begin{aligned}
& \mathcal{G}_{h}^{(r, m)}[w, z] \\
= & S_{0}^{(r, m)}[w] z^{(r+1, m)}+\sum_{i=1}^{n} S_{i .+}^{(r, m)}[w] z^{\left(r+1, m+e_{i}\right)}+\sum_{i=1}^{n} S_{i .-}^{(r, m)}[w] z^{\left(r+1, m-e_{i}\right)} \\
& +\sum_{(i, j) \in J_{+}^{(r, m)}[w]} \frac{1}{2 h_{i} h_{j}} f_{i j}\left(P^{(r, m)}[w]\right)\left[z^{\left(r+1, m+e_{i}+e_{j}\right)}+z^{\left(r+1, m-e_{i}-e_{j}\right)}\right] \\
& -\sum_{(i, j) \in J_{-}^{(r, m)}[w]} \frac{1}{2 h_{i} h_{j}} f_{i j}\left(P^{(r, m)}[w]\right)\left[z^{\left(r+1, m+e_{i}-e_{j}\right)}+z^{\left(r+1, m-e_{i}+e_{j}\right)}\right],
\end{aligned}
$$

where

$$
\begin{aligned}
& S_{0}^{(r, m)}[w]=\sum_{(i, j) \in J} \frac{1}{h_{i} h_{j}}\left|f_{i j}\left(P^{(r, m)}[w]\right)\right|-2 \sum_{i=1}^{n} \frac{1}{h_{i}^{2}} f_{i i}\left(P^{(r, m)}[w]\right), \\
& S_{i .+}^{(r, m)}[w]=\frac{1}{2 h_{i}} g_{i}\left(P^{(r, m)}[w]\right)+\frac{1}{h_{i}^{2}} f_{i i}\left(P^{(r, m)}[w]\right)-\sum_{\substack{j=1 \\
j \neq i}}^{n} \frac{1}{h_{i} h_{j}}\left|f_{i j}\left(P^{(r, m)}[w]\right)\right|,
\end{aligned}
$$


$S_{i .-}^{(r, m)}[w]=-\frac{1}{2 h_{i}} g_{i}\left(P^{(r, m)}[w]\right)+\frac{1}{h_{i}^{2}} f_{i i}\left(P^{(r, m)}[w]\right)-\sum_{\substack{j=1 \\ j \neq i}}^{n} \frac{1}{h_{i} h_{j}}\left|f_{i j}\left(P^{(r, m)}[w]\right)\right|$ and $S_{i .+}^{(r, m)}[w] \geq 0, S_{i .-}^{(r, m)}[w] \geq 0$ and $S_{0}^{(r, m)}[w]+\sum_{i=1}^{n} S_{i .+}^{(r, m)}[w]+\sum_{i=1}^{n} S_{i .-}^{(r, m)}[w]+\sum_{(i, j) \in J} \frac{1}{h_{i} h_{j}}\left|f_{i j}\left(P^{(r, m)}[w]\right)\right|=0$

Proof. The above lemma is a consequence of (1.7)-(1.10) and (2.7).

Theorem 2.2. Suppose that Assumption $\mathbf{H}[\mathbf{f}, \mathbf{g}, G]$ is satisfied and $\boldsymbol{h} \in \mathbb{H}$ and $0 \leq r \leq K-1$ is fixed.

1) If $v_{\boldsymbol{h}}:\left(E_{0 . \boldsymbol{h}} \cup E_{\boldsymbol{h}}\right) \cap\left(\left[-r_{0}, t^{(r+1)}\right] \times \mathbb{R}^{n}\right) \rightarrow \mathbb{R}$ and

$$
v_{\boldsymbol{h}}^{(r+1, m)} \leq h_{0} \mathcal{G}_{\boldsymbol{h}}^{(r, m)}\left[v_{\boldsymbol{h}}, v_{\boldsymbol{h}}\right]
$$

for $-(M-1) \leq m \leq M-1$ and $\Lambda_{\boldsymbol{h}}\left[v_{\boldsymbol{h}}\right]^{(r+1, m)} \leq 0$ on $\partial_{0} E_{\boldsymbol{h}}$, then $v_{h}^{(r+1, m)} \leq 0$ for $-M \leq m \leq M$.

2) If $v_{\boldsymbol{h}}:\left(E_{0 . \boldsymbol{h}} \cup E_{\boldsymbol{h}}\right) \cap\left(\left[-r_{0}, t^{(r+1)}\right] \times \mathbb{R}^{n}\right) \rightarrow \mathbb{R}$ and

$$
v_{\boldsymbol{h}}^{(r+1, m)} \geq h_{0} \mathcal{G}_{\boldsymbol{h}}^{(r, m)}\left[v_{\boldsymbol{h}}, v_{\boldsymbol{h}}\right]
$$

for $-(M-1) \leq m \leq M-1$ and $\Lambda_{\boldsymbol{h}}\left[v_{\boldsymbol{h}}\right]^{(r+1, m)} \geq 0$ on $\partial_{0} E_{\boldsymbol{h}}$, then $v_{h}^{(r+1, m)} \geq 0$ for $-M \leq m \leq M$.

Proof. Let us consider the first case. Let $\mu \in \mathbb{Z}^{n}$ be defined by relation

$$
v_{\mathbf{h}}^{(r+1, \mu)}=\max \left\{v_{\mathbf{h}}^{(r+1, m)}:\left(t^{(r+1)}, x^{(m)}\right) \in E_{0 . \mathbf{h}} \cup E_{\mathbf{h}}\right\} .
$$

Conversely, suppose that $v_{\mathbf{h}}^{(r+1, \mu)}>0$. Suppose that $-(M-1) \leq \mu \leq M-1$. It follows from (2.8) that

$$
\begin{aligned}
v_{\mathbf{h}}^{(r+1, m)} & \left(1-h_{0} S_{0}^{(r, m)}\left[v_{\mathbf{h}}\right]\right) \\
\leq & h_{0} \sum_{i=1}^{n} S_{i .+}^{(r, m)}\left[v_{\mathbf{h}}\right] v_{\mathbf{h}}^{\left(r+1, m+e_{i}\right)}+h_{0} \sum_{i=1}^{n} S_{i .-}^{(r, m)}\left[v_{\mathbf{h}}\right] v_{\mathbf{h}}^{\left(r+1, m-e_{i}\right)} \\
& +h_{0} \sum_{(i, j) \in J_{+}^{(r, m)}\left[v_{\mathbf{h}}\right]} \frac{1}{2 h_{i} h_{j}} f_{i j}\left(P^{(r, m)}\left[v_{\mathbf{h}}\right]\right)\left[v_{\mathbf{h}}^{\left(r+1, m+e_{i}+e_{j}\right)}+v_{\mathbf{h}}^{\left(r+1, m-e_{i}-e_{j}\right)}\right] \\
& -h_{0} \sum_{(i, j) \in J_{-}^{(r, m)}\left[v_{\mathbf{h}}\right]} \frac{1}{2 h_{i} h_{j}} f_{i j}\left(P^{(r, m)}\left[v_{\mathbf{h}}\right]\right)\left[v_{\mathbf{h}}^{\left(r+1, m+e_{i}-e_{j}\right)}+v_{\mathbf{h}}^{\left(r+1, m-e_{i}+e_{j}\right)}\right] .
\end{aligned}
$$

We see at once that $v_{\mathbf{h}}^{(r+1, \mu)} \leq 0$ for $-(M-1) \leq \mu \leq M-1$, which contradicts our assumption. Suppose that $\left(t^{(r+1)}, x^{(\mu)}\right) \in \partial_{0} E_{\mathbf{h}}$. We have 


$$
\begin{array}{ll}
v_{\mathbf{h}}^{(r+1, \mu)} \leq \frac{\gamma^{(r+1, \mu)}}{h_{i} \beta^{(r+1, \mu)}+\gamma^{(r+1, \mu)}} v_{\mathbf{h}}^{\left(r+1, \mu-e_{i}\right)} & \text { for }\left(t^{(r+1)}, x^{(\mu)}\right) \in \partial_{0} E_{\mathbf{h} . i}^{+}, \\
v_{\mathbf{h}}^{(r+1, \mu)} \leq \frac{\gamma^{(r+1, \mu)}}{h_{i} \beta^{(r+1, \mu)}+\gamma^{(r+1, \mu)}} v_{\mathbf{h}}^{\left(r+1, \mu+e_{i}\right)} & \text { for }\left(t^{(r+1)}, x^{(\mu)}\right) \in \partial_{0} E_{\mathbf{h} . i}^{-} .
\end{array}
$$

From this we conclude that $v_{\mathbf{h}}^{(r+1, \mu)} \leq 0$, which is impossible. The second case can be treated in a similar way.

TheOrem 2.3. Suppose that Assumption $\mathbf{H}[\mathbf{f}, \mathbf{g}, G]$ is satisfied and $\psi_{\boldsymbol{h}}$ : $E_{0 . \boldsymbol{h}} \rightarrow \mathbb{R}, \Psi_{\boldsymbol{h}}: \partial_{0} E_{\boldsymbol{h}} \rightarrow \mathbb{R}$. Then there is exactly one solution $u_{\boldsymbol{h}}: E_{0 . \boldsymbol{h}} \cup$ $E_{\boldsymbol{h}} \rightarrow \mathbb{R}$ of problem (1.5), (1.11), (1.12).

Proof. Suppose that $0 \leq r<K$ is fixed and that the solution $u_{\mathbf{h}}$ to problem (1.5), (1.11), (1.12) is given on the set $\left(E_{0 . \mathbf{h}} \cup E_{\mathbf{h}}\right) \cap\left(\left[-b_{0}, t^{(r)}\right] \times \mathbb{R}^{n}\right)$. We prove that the values $u_{\mathbf{h}}^{(r+1, m)},-M \leq m \leq M$ exist, and that they are unique. It is sufficient to show that there exists exactly one solution of the system of equations

$$
z^{(r+1, m)}=u_{\mathbf{h}}^{(r, m)}+h_{0} \mathcal{G}_{\mathbf{h}}^{(r, m)}\left[u_{\mathbf{h}}, z_{\mathbf{h}}\right]+h_{0} G\left(P^{(r, m)}\left[u_{\mathbf{h}}\right]\right)
$$

where $-(M-1) \leq m \leq M-1$, and

$$
\Lambda[z]^{(r+1, m)}=\Psi_{\mathbf{h}}^{(r+1, m)} \text { on } \partial_{0} E_{\mathbf{h}} .
$$

Consider problem

$$
\begin{gathered}
z^{(r+1, m)}=h_{0} \mathcal{G}_{\mathbf{h}}^{(r, m)}\left[u_{\mathbf{h}}, z_{\mathbf{h}}\right], \quad \text { where }-(M-1) \leq m \leq M-1, \\
\Lambda[z]^{(r+1, m)}=0 \quad \text { on } \partial_{0} E_{\mathbf{h}} .
\end{gathered}
$$

We conclude from Theorem (2.2), that above problem has exactly one solution, which proves the theorem.

Assumption $\mathbf{H}\left[T_{\mathbf{h}}\right]$. The operator $T_{\mathbf{h}}: \mathcal{F}\left(B_{\mathbf{h}}, \mathbb{R}\right) \rightarrow C(B, \mathbb{R})$ satisfies the conditions:

1) for $w, \tilde{w} \in \mathcal{F}\left(B_{\mathbf{h}}, \mathbb{R}\right)$ we have

$$
\left\|T_{\mathbf{h}}[w]-T_{\mathbf{h}}[\tilde{w}]\right\|_{B} \leq\|w-\tilde{w}\|_{B_{\mathbf{h}}},
$$

2) if $w: B \rightarrow \mathbb{R}$ is of class $C^{1}$ then there is $\gamma_{*}: \mathbb{H} \rightarrow \mathbb{R}_{+}$such that

$$
\left\|T_{\mathbf{h}}\left[w_{\mathbf{h}}\right]-w\right\|_{B} \leq \gamma_{*}(\mathbf{h}), \text { and } \lim _{\mathbf{h} \rightarrow 0} \gamma_{*}(\mathbf{h})=0
$$

where $w_{\mathbf{h}}$ is the restriction of $w$ to the set $B_{\mathbf{h}}$.,

3) if $\mathbf{O}_{\mathbf{h}} \in \mathcal{F}\left(E_{0 . \mathbf{h}} \cup E_{\mathbf{h}}, \mathbb{R}\right)$ is given by $\mathbf{O}_{\mathbf{h}}(t, x)=0$ for $(t, x) \in E_{0 . \mathbf{h}} \cup E_{\mathbf{h}}$ then $T_{\mathbf{h}}\left[\mathbf{O}_{\mathbf{h}}\right](t, x)=0$ for $(t, x) \in E_{0} \cup E$. 
Lemma 2.2. Suppose that Assumptions $\mathbf{H}\left[T_{\mathbf{h}}\right], \mathbf{H}[\mathbf{f}, \mathbf{g}, G]$ are satisfied and $v_{\boldsymbol{h}}$ is a solution of problem (1.5), (1.11), (1.12) and

$$
\left|\psi_{\boldsymbol{h}}^{(r, m)}\right| \leq \tilde{\eta} \text { on } E_{0 . \boldsymbol{h}} \text { and }\left|\Psi_{\boldsymbol{h}}^{(r, m)}\right| \leq \tilde{B} \omega\left(t^{(r)}, \tilde{\eta}\right) \quad \text { on } \partial_{0} E_{\boldsymbol{h}} .
$$

Then

$$
\left|v_{\boldsymbol{h}}^{(r, m)}\right| \leq \omega\left(t^{(r)}, \tilde{\eta}\right) \quad \text { on } E_{0 . \boldsymbol{h}} \cup E_{\boldsymbol{h}} .
$$

Proof. Write

$$
\varepsilon_{\mathbf{h}}^{(r)}=\max \left\{\left|v_{\mathbf{h}}^{(i, m)}\right|:\left(t^{(i)}, x^{(m)}\right) \in E_{0 . \mathbf{h}} \cup E_{\mathbf{h}}, i \leq r\right\}, \quad 0 \leq r \leq K .
$$

It is sufficient to show that

$$
\varepsilon_{\mathbf{h}}^{(r)} \leq \omega\left(t^{(r)}, \tilde{\eta}\right)
$$

where $0 \leq r \leq K$. From (2.11) we deduce that (2.13) is true for $r=0$. Assuming that $\varepsilon_{\mathbf{h}}^{(j)} \leq \omega\left(t^{(j)}, \tilde{\eta}\right)$ for $0 \leq j \leq r-1$ we will prove (2.13) for $r$. There exists $\left(t^{(i)}, x^{(m)}\right) \in E_{\mathbf{h}}$ such that $\varepsilon_{\mathbf{h}}^{(r)}=\left|v_{\mathbf{h}}^{(i, m)}\right|$. If $i<r$ then $\left|v_{\mathbf{h}}^{(i, m)}\right| \leq \varepsilon_{\mathbf{h}}^{(r-1)} \leq \omega\left(t^{(r-1)}, \tilde{\eta}\right)$ and consequently $\varepsilon_{\mathbf{h}}^{(r)} \leq \omega\left(t^{(r)}, \tilde{\eta}\right)$. Suppose that $\varepsilon_{\mathbf{h}}^{(r)}=\left|v_{\mathbf{h}}^{(r, m)}\right|$. Then two possibilities can happen, either $\varepsilon_{\mathbf{h}}^{(r)}=v_{\mathbf{h}}^{(r, m)}$ or $\varepsilon_{\mathbf{h}}^{(r)}=-v_{\mathbf{h}}^{(r, m)}$. Let us consider the first case. It follows form (2.11) that for $\left(t^{(r)}, x^{(m)}\right) \in \partial_{0} E_{\mathbf{h}}$ we have

$$
\tilde{B} v_{\mathbf{h}}^{(r, m)} \leq\left|\Psi_{\mathbf{h}}^{(r, m)}\right| \leq \tilde{B} \omega\left(t^{(r)}, \tilde{\eta}\right) .
$$

Hence $\varepsilon_{\mathbf{h}}^{(r)} \leq \omega\left(t^{(r)}, \tilde{\eta}\right)$. Suppose that $\left(t^{(r)}, x^{(m)}\right) \in E_{\mathbf{h}}$. It follows from (1.6) and (2.8) that

$$
\begin{aligned}
v_{\mathbf{h}}^{(r, m)}( & \left.1-h_{0} S_{0}^{(r-1, m)}\left[v_{\mathbf{h}}\right]\right) \\
= & v_{\mathbf{h}}^{(r-1, m)}+\left[h_{0} \sum_{i=1}^{n} S_{i .+}^{(r-1, m)}\left[v_{\mathbf{h}}\right] v_{\mathbf{h}}^{\left(r, m+e_{i}\right)}+h_{0} \sum_{i=1}^{n} S_{i .-}^{(r-1, m)}\left[v_{\mathbf{h}}\right] v_{\mathbf{h}}^{\left(r, m-e_{i}\right)}\right. \\
& +h_{0} \sum_{(i, j) \in J_{+}^{(r-1, m)}\left[v_{\mathbf{h}}\right]} \frac{1}{2 h_{i} h_{j}} f_{i j}\left(P^{(r-1, m)}\left[v_{\mathbf{h}}\right]\right)\left[v_{\mathbf{h}}^{\left(r, m+e_{i}+e_{j}\right)}+v_{\mathbf{h}}^{\left(r, m-e_{i}-e_{j}\right)}\right] \\
& \left.-h_{0} \sum_{(i, j) \in J_{-}^{(r-1, m)}\left[v_{\mathbf{h}}\right]} \frac{1}{2 h_{i} h_{j}} f_{i j}\left(P^{(r-1, m)}\left[v_{\mathbf{h}}\right]\right)\left[v_{\mathbf{h}}^{\left(r, m-e_{i}+e_{j}\right)}+v_{\mathbf{h}}^{\left(r, m+e_{i}-e_{j}\right)}\right]\right] \\
& +h_{0} G\left(P^{(r-1, m)}\left[v_{\mathbf{h}}\right]\right) .
\end{aligned}
$$

It follows from induction and from condition 1) of Assumption $\mathbf{H}[\mathbf{f}, \mathbf{g}, G]$ that

$$
\varepsilon_{\mathbf{h}}^{(r)} \leq \varepsilon_{\mathbf{h}}^{(r-1)}+h_{0} \varrho\left(t^{(r-1)}, \varepsilon_{\mathbf{h}}^{(r-1)}\right)
$$


and consequently

$$
\varepsilon_{\mathbf{h}}^{(r)} \leq \omega\left(t^{(r)}, \tilde{\eta}\right)
$$

The case $\varepsilon_{\mathbf{h}}^{(r)}=-v_{\mathbf{h}}^{(r, m)}$ can be treated in a similar way. Hence, the proof of (2.13) is completed by induction.

\section{Convergence of difference schemes}

Write $A=\omega(a, \tilde{\eta})$, where $\omega(\cdot, \tilde{\eta})$ is the maximal solution to (2.1) and $K_{C(B, \mathbb{R})}(A)=\left\{w \in C(B, \mathbb{R}):\|w\|_{B} \leq A\right\}$.

Assumption $\mathbf{H}[\sigma]$. The function $\sigma:[0, a] \times \mathbb{R}_{+} \rightarrow \mathbb{R}_{+}$is continuous, it is nondecreasing with respect to both variables and for each $L \geq 1$ the maximal solution of the Cauchy problem

$$
\omega^{\prime}(t)=L \sigma(t, \omega(t)), \quad \omega(0)=0
$$

is $\tilde{\omega}(t)=0$ for $t \in[0, a]$.

Assumption A[f, g, $G]$. The functions $\mathbf{f}: \Xi \rightarrow M_{n \times n}, \mathbf{g}: \Xi \rightarrow \mathbb{R}^{n}$, $G: \Xi \rightarrow \mathbb{R}$ satisfy Assumption $\mathbf{H}[\mathbf{f}, \mathbf{g}, G]$ and there is $\sigma:[0, a] \times \mathbb{R}_{+} \rightarrow \mathbb{R}_{+}$ such that Assumption $\mathbf{H}[\sigma]$ is satisfied and

$$
\begin{aligned}
\|\mathbf{f}(t, x, w)-\mathbf{f}(t, x, \bar{w})\|_{[n \times n]} & \leq \sigma\left(t,\|w-\bar{w}\|_{B}\right), \\
\|\mathbf{g}(t, x, w)-\mathbf{g}(t, x, \bar{w})\|_{[n]} & \leq \sigma\left(t,\|w-\bar{w}\|_{B}\right), \\
|G(t, x, w)-G(t, x, \bar{w})| & \leq \sigma\left(t,\|w-\bar{w}\|_{B}\right),
\end{aligned}
$$

where $(t, x) \in E, w, \bar{w} \in K_{C(B, \mathbb{R})}(A)$.

REMARK 3.1. Note that we have assumed estimates (3.1)-(3.3) for $w, \bar{w} \in$ $K_{C(B, \mathbb{R})}[A]$ only. It is clear, that there are differential equations with deviated variables and differential integral equations such that corresponding functions $\mathbf{f}$, $\mathbf{g}$ and $G$ satisfy conditions (3.1)-(3.3), respectively on $E \times$ $K_{C(B, \mathbb{R})}(A)$ and they do not satisfy (3.1)-(3.3) on $\Xi$.

TheOREm 3.1. Suppose that Assumptions $\mathbf{A}[\mathbf{f}, \mathbf{g}, G], \mathbf{H}\left[T_{\mathbf{h}}\right]$ are satisfied and

1) $u_{\boldsymbol{h}}: E_{0 . \boldsymbol{h}} \cup E_{\boldsymbol{h}} \rightarrow \mathbb{R}$ is a solution of (1.5), (1.11), (1.12),

2) $v: E_{0} \cup E \rightarrow \mathbb{R}$ is a solution of (1.1)-(1.4) and $v$ is of class $C_{*}$ and $v_{\boldsymbol{h}}$ is restriction of $v$ to the set $E_{0 . h} \cup E_{h}$,

3) for $\alpha_{0}: \mathbb{H} \rightarrow \mathbb{R}_{+}$the following initial boundary inequalities are satisfied $\left|\psi_{\boldsymbol{h}}^{(r, m)}-\psi^{(r, m)}\right| \leq \alpha_{0}(\boldsymbol{h}) \quad$ on $E_{0 . \boldsymbol{h}}, \quad\left|\Psi_{\boldsymbol{h}}^{(r, m)}-\Psi^{(r, m)}\right| \leq h_{0} \alpha_{0}(\boldsymbol{h}) \quad$ on $\partial_{0} E_{\boldsymbol{h}}$ and $\lim _{\boldsymbol{h} \rightarrow 0} \alpha_{0}(\boldsymbol{h})=0$,

4) there is $c_{0}>0$ such that $h_{i} \leq c_{0} h_{0}$ for $i=1, \ldots, n$. 
Then there is $\alpha: \mathbb{H} \rightarrow \mathbb{R}_{+}$such that

$$
\left|\left(u_{\boldsymbol{h}}-v_{\boldsymbol{h}}\right)^{(r, m)}\right| \leq \alpha(\boldsymbol{h}) \quad \text { on } E_{\boldsymbol{h}}
$$

and $\lim _{\boldsymbol{h} \rightarrow 0} \alpha(\boldsymbol{h})=0$.

Proof. Our proof starts with the above observation that

$$
\begin{aligned}
\delta_{i j} v^{(r, m)}= & \frac{1}{2} \int_{0}^{1} \int_{0}^{1} \partial_{x_{i} x_{j}} v\left(t^{(r)}, x^{(m)}+s h_{i} e_{i}+\tau h_{j} e_{j}\right) d s d \tau \\
& +\frac{1}{2} \iint_{0}^{1} \partial_{x_{i} x_{j}} v\left(t^{(r)}, x^{(m)}-s h_{i} e_{i}-\tau h_{j} e_{j}\right) d s d \tau \quad \text { for }(i, j) \in J_{+}, \\
\delta_{i j} v^{(r, m)}= & \frac{1}{2} \int_{0}^{1} \int_{0}^{1} \partial_{x_{i} x_{j}} v\left(t^{(r)}, x^{(m)}+s h_{i} e_{i}-\tau h_{j} e_{j}\right) d s d \tau \\
& +\frac{1}{2} \int_{0}^{1} \int_{0}^{1} \partial_{x_{i} x_{j}} v\left(t^{(r)}, x^{(m)}-s h_{i} e_{i}+\tau h_{j} e_{j}\right) d s d \tau \quad \text { for }(i, j) \in J_{-} .
\end{aligned}
$$

It follows that there are $\Gamma_{\mathbf{h}}: E_{\mathbf{h}} \rightarrow \mathbb{R}, \gamma_{1}: \mathbb{H} \rightarrow \mathbb{R}_{+}$such that

$$
\begin{array}{ll}
\delta_{0} v_{\mathbf{h}}^{(r, m)}=F_{\mathbf{h}}\left[v_{\mathbf{h}}\right]^{(r, m)}+\Gamma_{\mathbf{h}}^{(r, m)} & \text { on } E_{\mathbf{h}}, \\
\delta_{i}^{+} v_{\mathbf{h}}^{(r, m)}=\partial_{x_{i}} v^{(r, m)}+\Gamma_{\mathbf{h}}^{(r, m)} & \text { on } \partial_{0} E_{\mathbf{h} . i}^{+}, \\
\delta_{i}^{-} v_{\mathbf{h}}^{(r, m)}=\partial_{x_{i}} v^{(r, m)}-\Gamma_{\mathbf{h}}^{(r, m)} & \text { on } \partial_{0} E_{\mathbf{h} . i}^{-},
\end{array}
$$

where $i=1, \ldots, n$ and

$$
\left|\Gamma_{\mathbf{h}}^{(r, m)}\right| \leq \gamma_{1}(\mathbf{h}) \quad \text { on } E_{\mathbf{h}} \quad \text { and } \quad \lim _{\mathbf{h} \rightarrow 0} \gamma_{1}(\mathbf{h})=0 .
$$

Write $z_{\mathbf{h}}=u_{\mathbf{h}}-v_{\mathbf{h}}$ and

$$
\varepsilon_{\mathbf{h}}^{(r)}=\max \left\{\left|z_{\mathbf{h}}^{(i, m)}\right|:\left(t^{(i)}, x^{(m)}\right) \in E_{0 . \mathbf{h}} \cup E_{\mathbf{h}}, i \leq r\right\}, \quad 0 \leq r \leq K .
$$

We prove that the function $\varepsilon_{\mathbf{h}}^{(r)}$ satisfies the recurrent inequality

$$
\varepsilon_{\mathbf{h}}^{(r+1)} \leq \varepsilon_{\mathbf{h}}^{(r)}+h_{0} L \sigma\left(t^{(r)}, \varepsilon_{\mathbf{h}}^{(r)}\right)+h_{0} \tilde{\gamma}(\mathbf{h}), \quad 0 \leq r \leq K-1,
$$

where $L \geq 1$ and $\tilde{\gamma}(\mathbf{h})=\gamma_{1}(\mathbf{h})\left(1+c_{0}\right)+\tilde{B}^{-1} \alpha_{0}(\mathbf{h})$. Suppose that $\left(t^{(r+1)}, x^{(m)}\right)$ $\in E_{\mathbf{h}}$ and $-(M-1) \leq m \leq M-1$. Then we have

$$
z_{\mathbf{h}}^{(r+1, m)}=z_{\mathbf{h}}^{(r, m)}+h_{0}\left[F_{\mathbf{h}}\left[u_{\mathbf{h}}\right]^{(r, m)}-F_{\mathbf{h}}\left[v_{\mathbf{h}}\right]^{(r, m)}\right] .
$$

It follows from (2.8) that 


$$
\begin{aligned}
z_{\mathbf{h}}^{(r+1, m)} & \left(1-h_{0} S_{0}^{(r, m)}\left[u_{\mathbf{h}}\right]\right) \\
= & z_{\mathbf{h}}^{(r, m)}+h_{0} \sum_{i=1}^{n} S_{i .+}^{(r, m)}\left[u_{\mathbf{h}}\right] z_{\mathbf{h}}^{\left(r+1, m+e_{i}\right)} \\
& +h_{0} \sum_{i=1}^{n} S_{i .-}^{(r, m)}\left[u_{\mathbf{h}}\right] z_{\mathbf{h}}^{\left(r+1, m-e_{i}\right)} \\
& -h_{0} \sum_{(i, j) \in J_{-}^{(r, m)}\left[u_{\mathbf{h}}\right]} \frac{1}{2 h_{i} h_{j}} f_{i j}\left(P^{(r, m)}\left[u_{\mathbf{h}}\right]\right)\left[z_{\mathbf{h}}^{\left(r+1, m+e_{i}-e_{j}\right)}+z_{\mathbf{h}}^{\left(r+1, m-e_{i}+e_{j}\right)}\right] \\
& +h_{0} \sum_{(i, j) \in J_{+}^{(r, m)}\left[u_{\mathbf{h}}\right]}^{2 h_{i} h_{j}} f_{i j}\left(P^{(r, m)}\left[u_{\mathbf{h}}\right]\right)\left[z_{\mathbf{h}}^{\left(r+1, m+e_{i}+e_{j}\right)}+z_{\mathbf{h}}^{\left(r+1, m-e_{i}-e_{j}\right)}\right] \\
& +h_{0}\left[G\left(P^{(r, m)}\left[u_{\mathbf{h}}\right]\right)-G\left(P^{(r, m)}\left[v_{\mathbf{h}}\right]\right)\right]-h_{0} \Gamma_{\mathbf{h}}^{(r, m)} \\
& +h_{0} \sum_{i, j=1}^{n}\left[f_{i j}\left(P^{(r, m)}\left[u_{\mathbf{h}}\right]\right)-f_{i j}\left(P^{(r, m)}\left[v_{\mathbf{h}}\right]\right)\right] \delta_{i j} v_{\mathbf{h}}^{(r+1, m)} \\
& +h_{0} \sum_{i=1}^{n}\left[g_{i}\left(P^{(r, m)}\left[u_{\mathbf{h}}\right]\right)-g_{i}\left(P^{(r, m)}\left[v_{\mathbf{h}}\right]\right)\right] \delta_{i} v_{\mathbf{h}}^{(r+1, m)} .
\end{aligned}
$$

There is $\tilde{c} \in \mathbb{R}_{+}$such that

$$
\left|\delta_{i} v_{\mathbf{h}}^{(r+1, m)}\right| \leq \tilde{c}, i=1, \ldots, n, \quad\left|\delta_{i j} v_{\mathbf{h}}^{(r+1, m)}\right| \leq \tilde{c}, i, j=1, \ldots, n .
$$

It follows from Assumptions $\mathbf{A}[\mathbf{f}, \mathbf{g}, G], \mathbf{H}\left[T_{\mathbf{h}}\right]$ and (3.8) that

$$
\left|z_{\mathbf{h}}^{(r+1, m)}\right| \leq \varepsilon_{\mathbf{h}}^{(r)}+h_{0}(2 \tilde{c}+1) \sigma\left(t^{(r)},\left(\varepsilon_{\mathbf{h}}\right)_{t^{(r)}}\right)+h_{0} \gamma_{1}(\mathbf{h}) .
$$

Suppose that $\left(t^{(r+1)}, x^{(m)}\right) \in \partial_{0} E_{\mathbf{h} . i}^{+}$. Then we have

$$
\begin{aligned}
z_{\mathbf{h}}^{(r+1, m)}= & \frac{\gamma^{(r+1, m)}}{h_{i} \beta^{(r+1, m)}+\gamma^{(r+1, m)}} z_{\mathbf{h}}^{\left(r+1, m-e_{i}\right)} \\
& +\frac{\gamma^{(r+1, m)}}{h_{i} \beta^{(r+1, m)}-\gamma^{(r+1, m)}} h_{i} \Gamma_{\mathbf{h}}^{(r+1, m)} \\
& +\frac{h_{i}}{h_{i} \beta^{(r+1, m)}+\gamma^{(r+1, m)}}\left[\Psi_{\mathbf{h}}^{(r+1, m)}-\Psi^{(r+1, m)}\right]
\end{aligned}
$$

and consequently

$(3.10)\left|z_{\mathbf{h}}^{(r+1, m)}\right| \leq \varepsilon_{\mathbf{h}}^{(r)}+h_{0} L \sigma\left(t^{(r)}, \varepsilon_{\mathbf{h}}^{(r)}\right)+h_{0} \gamma_{1}(\mathbf{h})+c_{0} h_{0} \gamma_{1}(\mathbf{h})+\frac{1}{\tilde{B}} h_{0} \alpha_{0}(\mathbf{h})$.

In a similar way we prove $(3.10)$ for $\left(t^{(r+1)}, x^{(m)}\right) \in \partial_{0} E_{\mathbf{h} . i}^{-}$. Estimates (3.9) and (3.10) imply (3.7). Lets us consider the Cauchy problem 


$$
\omega^{\prime}(t)=L \sigma(t, \omega(t))+\tilde{\gamma}(\mathbf{h}), \quad \omega(0)=\alpha_{0}(\mathbf{h}) .
$$

There is $\varepsilon_{0}>0$ such that the maximal solution $\omega(\cdot, \mathbf{h})$ to $(3.11)$ is defined on $[0, a]$ for $0 \leq\|\mathbf{h}\|<\varepsilon_{0}$ and $\lim _{\mathbf{h} \rightarrow 0} \omega(t, \mathbf{h})=0$ uniformly on $[0, a]$. We conclude from Assumption $\mathbf{H}[\sigma]$ that

$$
\omega\left(t^{(r+1)}, \mathbf{h}\right) \geq \omega\left(t^{(r)}, \mathbf{h}\right)+h_{0} L \sigma\left(t^{(r)}, \omega\left(t^{(r)}, \mathbf{h}\right)\right)+h_{0} \tilde{\gamma}(\mathbf{h}), 0 \leq r \leq K-1 .
$$

This gives

$$
\varepsilon_{\mathbf{h}}^{(r)} \leq \omega\left(t^{(r)}, \mathbf{h}\right) \quad \text { for } 0 \leq r \leq K .
$$

Then assertion (3.4) is satisfied with $\alpha(\mathbf{h})=\omega(a, \mathbf{h})$.

REMARK 3.2. Suppose that all the assumptions of Theorem 3.1 are satisfied and $\sigma:[0, a] \times \mathbb{R}_{+} \times C\left(I, \mathbb{R}_{+}\right) \rightarrow \mathbb{R}_{+}$is given by

$$
\sigma(t, p)=\tilde{L} p \quad \text { on }[0, a] \times \mathbb{R}_{+},
$$

where $\tilde{L} \in \mathbb{R}_{+}$. Then

$$
\left|u_{\mathbf{h}}^{(r, m)}-v_{\mathbf{h}}^{(r, m)}\right| \leq \tilde{\alpha}(\mathbf{h}) \quad \text { on } E_{\mathbf{h}}
$$

where

$$
\begin{aligned}
& \tilde{\alpha}(\mathbf{h})=\alpha_{0}(\mathbf{h}) e^{\tilde{L} a}+\frac{\tilde{\gamma}(\mathbf{h})}{\tilde{L}}\left(e^{\tilde{L} a}-1\right) \quad \text { if } \tilde{L}>0, \\
& \tilde{\alpha}(\mathbf{h})=\alpha_{0}(\mathbf{h})+a \tilde{\gamma}(\mathbf{h}) \quad \text { if } \tilde{L}=0 .
\end{aligned}
$$

We obtain the above estimates by solving problem (3.11) with $\sigma(t, p)=\tilde{L} p$.

\section{Numerical examples}

$E_{0}=\{0\} \times[-1,1] \times[-1,1], E=[0,0.5] \times[-1,1] \times[-1,1] . \quad$ Initialboundary problems considered in the present section have solutions on $E$.

EXAMPLE 4.1. Consider the differential equation with deviated variables

$$
\begin{aligned}
& \partial_{t} z(t, x, y) \\
&=\left\{2+\sin \left[e^{\frac{x}{2}} z\left(t, \frac{x-1}{2}, \frac{y-1}{2}\right)-e^{\frac{y}{2}} z(t, x, y)\right]\right\} \partial_{x x} z(t, x, y) \\
&+\left\{2+\cos \left[e^{\frac{x}{2}} z\left(t, \frac{x+1}{2}, \frac{y+1}{2}\right)-e^{\frac{y}{2}} z(t, x, y)\right]\right\} \partial_{y y} z(t, x, y) \\
&+\sin \left[z\left(t, \frac{x}{2}, y\right)+z\left(t, x, \frac{y}{2}\right)-\left(e^{-\frac{x}{2}}+e^{\frac{y}{2}}\right) z(t, x, y)\right] \partial_{x y} z(t, x, y) \\
&-5 z(t, x, y)+e^{x-y},
\end{aligned}
$$

and the initial-boundary conditions

$$
z(0, x, y)=0, \quad(x, y) \in[-1,1] \times[-1,1]
$$


and

$$
\begin{aligned}
z(t, 1, y)+\partial_{x} z(t, 1, y) & =2 t e^{1-y}, \\
z(t,-1, y)-\partial_{x} z(t,-1, y) & =0, \quad y \in[-1,1], \\
z(t, x, 1)+\partial_{y} z(t, x, 1) & =0, \\
z(t, x,-1)-\partial_{y} z(t, x,-1) & =2 t e^{x+1}, \quad x \in[-1,1] .
\end{aligned}
$$

The solution of (4.1-4.3) is known, it is $v(t, x, y)=t e^{x-y}$. Let us denote by $u_{\mathbf{h}}: E_{\mathbf{h}} \rightarrow \mathbb{R}$ the solution of implicit difference problem corresponding to (4.1)-(4.3). Write

$$
\varepsilon_{\mathbf{h}}^{(r)}=\frac{1}{\left(2 N_{1}+1\right)\left(2 N_{2}+1\right)} \sum_{m \in M}\left|u_{\mathbf{h}}^{(r, m)}-v_{\mathbf{h}}^{(r, m)}\right|, \quad 0 \leq r \leq N_{0},
$$

where $v_{\mathbf{h}}$ is the restriction of $v$ to the set $E_{\mathbf{h}}$ and $M=\left\{m \in\left(m_{1}, m_{2}\right)\right.$ : $\left.-N_{1} \leq m_{1} \leq N_{1},-N_{2} \leq m_{2} \leq N_{2}\right\}$ and $N_{0} h_{0}=0.5, N_{1} h_{1}=0.5, N_{2} h_{2}=$ 0.5. The numbers $\varepsilon_{\mathbf{h}}^{(r)}$ are the arithmetical means of the errors with fixed $t^{(r)}$. We give experimental values of the above defined errors for $h_{0}=0.001$, $h_{1}=h_{2}=0.004$.

\section{Table I}

$\begin{array}{llllllll}t^{(r)}: & 0.07 & 0.14 & 0.21 & 0.28 & 0.35 & 0.42 & 0.49 \\ \varepsilon_{h}^{(r)}: & 0.0003808 & 0.0006355 & 0.0008261 & 0.0009835 & 0.0011245 & 0.0012578 & 0.0013883\end{array}$

We have solved numerically problem (4.1-4.3) by using explicit difference schemes for $h_{0}=0.001, h_{1}=h_{2}=0.004$. In this case condition (1.13) is not satisfied and errors exceeded $12 \cdot 10^{16}$ for $t^{(r)}=0.01$.

EXAMPle 4.2. Consider the differential integral equation

$$
\begin{aligned}
\partial_{t} z(t, x, y)= & \left\{2+\cos \left[\int_{0}^{x} z(t, s, y) d s-z(t, x, y)\right]\right\} \partial_{x x} z(t, x, y) \\
& +\left\{2-\cos \left[\int_{0}^{y} z(t, x, s) d s+z(t, x, y)\right]\right\} \partial_{y y} z(t, x, y) \\
& +\cos \left[\int_{0}^{y} \int_{0}^{x} z(t, s, r) d s d r+z(t, x, y)\right] \partial_{x y} z(t, x, y) \\
& -f(t, x, y) z(t, x, y)+g(t, x, y)
\end{aligned}
$$

where

$$
\begin{aligned}
f(t, x, y)= & 4+\cos \left[\left(e^{t}-1\right) e^{-y}\right]-\cos \left[\left(e^{t}-1\right) e^{x}\right] \\
& -\cos \left[\left(e^{t}-1\right) e^{x}+\left(e^{t}-1\right) e^{-y}-e^{t}+1\right], \\
g(t, x, y)= & e^{t} e^{x-y}
\end{aligned}
$$


with initial-boundary conditions

$$
z(0, x, y)=0 \quad \text { for }(x, y) \in[-1,1] \times[-1,1],
$$

and

$(t, y) \in[0,0.5] \times[-1,1]$

$$
z(t, x, 1)+\partial_{y} z(t, x, 1)=0, \quad z(t, x,-1)-\partial_{y} z(t, x,-1)=2\left(e^{t}-1\right) e^{x+1},
$$

$(t, x) \in[0,0.5] \times[-1,1]$. The solution of $(4.5)-(4.7)$ is known, it is $z(t, x, y)=$ $\left(e^{t}-1\right) e^{x-y}$.

Let us denote by $u_{\mathbf{h}}: E_{\mathbf{h}} \rightarrow \mathbb{R}$, the solution of implicit difference problem corresponding to (4.5)-(4.7). Let $\varepsilon_{\mathbf{h}}^{(r)}$ be the arithmetical means of the errors defined by (4.4). In Table II, we give experimental values of $\varepsilon_{\mathbf{h}}^{(r)}$ for $h_{0}=$ $0.001, h_{1}=h_{2}=0.004$.

\section{Table II}

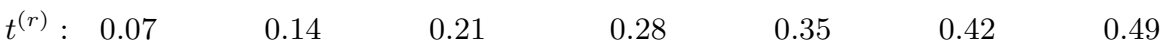

$\begin{array}{llllllll}\varepsilon_{h}^{(r)}: & 0.0002246 & 0.0004542 & 0.00073299 & 0.0013553 & 0.0025644 & 0.0042563 & 0.00694559\end{array}$

We have solved numerically problem (4.1-4.3) by using explicit difference schemes for $h_{0}=0.001, h_{1}=h_{2}=0.004$. In this case, condition (1.13) is not satisfied and errors exceeded $44 \cdot 10^{15}$ for $t^{(r)}=0.01$.

The results presented in the paper show that there are implicit difference methods for (1.1)-(1.4), which are convergent and the corresponding explicit difference schemes are not convergent. Note that our results are new also in the case of quasilinear differential equations without the functional dependence.

\section{References}

[1] R. Ciarski, Stability of difference equations generated by parabolic differential functional equations, Demonstratio Math. 38 (2005), 101-117.

[2] R. Ciarski, Numerical approximations of parabolic differential functional equations with the initial boundary conditions of the Neumann type, Ann. Polon. Mat. 84(2) (2004), 103-119.

[3] W. Czernous, Z. Kamont, Implicit difference methods for parabolic functional differential equations, ZAMM Z. Angew. Math. Mech. 85(5) (2005), 326-338.

[4] Z. Kamont, Hyperbolic Functional Differential Inequalities and Applications, Kluwer Academic Publishers, Dordrecht, 1999.

[5] K. Kropielnicka, Implicit difference methods for quasilinear parabolic functional differential systems, Univ. Iagel. Acta Math. 45 (2007), 175-195. 
[6] K. Kropielnicka, Implicit difference methods for parabolic functional differential problems of the Neumann type, Nonl. Oscill. 11 (2008), 65-80.

[7] Lu Xin, Monotone method and convergence acceleration for finite-difference solutions of parabolic problems with time delays, Numer. Methods Partial Differential Equations 11(6) (1995), 591-602.

[8] Lu Xin, Combined iterative methods for numerical solutions of parabolic problems with time delays, Appl. Math. Comput. 89(1-3) (1998), 213-224.

[9] C. V. Pao, Finite difference reaction-diffusion systems with coupled boundary conditions and time delays, J. Math. Anal. Appl. 272(2) (2002), 407-434.

[10] C. V. Pao, Finite difference solutions of reaction diffusion with continuous time delays, Comput. Math. Appl. 42(3-5) (2001), 399-412.

[11] A. A. Samarskii, The Theory of Difference Schemes, Marcel Dekker, Inc., New York, 2001.

[12] A. A. Samarskii, P. P. Matus, P. N. Vabishchevich, Difference Schemes with Operator Factors and its Applications, Kluwer Academic Publishers, Dordrecht, 2002.

[13] L. Sapa, A finite difference method for quasi-linear and nonlinear differential functional parabolic equations with Dirichlet's condition, Ann. Polon. Math. 93(2) (2008), 113-133.

[14] L. Sapa, A finite difference method for quasi-linear and nonlinear differential functional parabolic equations with Neumann's condition, Comment. Math. 1 (2009), 83106.

[15] Y. M. Wang, C. V. Pao, Time-delayed finite difference reaction-diffusion systems with nonquasimonotone functions, Numer. Math. 103(3) (2006), 485-513.

\author{
INSTITUTE OF MATHEMATICS \\ UNIVERSITY OF GDAŃSK \\ Wit Stwosz Street 57 \\ 80-952 GDAŃSK, POLAND \\ E-mail: milena.matusik@math.ug.edu.pl
}

Received November 10, 2010. 